\title{
Plasmonic quasicrystals with broadband transmission enhancement
}

SUBJECT AREAS:

METAMATERIALS

NANOSCALE MATERIALS

LITHOGRAPHY

NANOPHOTONICS AND

PLASMONICS

Received

16 October 2013

Accepted

22 May 2014

Published

11 June 2014

Correspondence and requests for materials should be addressed to V.G.A. (achanta@tifr. res.in)

\footnotetext{
* Current address:

Centre for Quantum

Dynamics, Griffith

University, Nathan

QLD 4111 Australia.
}

\author{
Sachin Kasture*, Ajith P. Ravishankar, V. J. Yallapragada, Raj Patil, Nikesh V. Valappil, Gajendra Mulay \\ \& Venu Gopal Achanta
}

DCMPMS, Tata Institute of Fundamental Research, Homi Bhabha Road, Mumbai 400005 INDIA. long range ordering but lack translational symmetry) and surface plasmon polariton mediated effects, offer several advantages over plasmonic crystals (PICs). For example, in PlQCs one could have broadband, polarization independent response. However, large area patterning by electron beam lithography requires precise lattice coordinates as well as a practical way to design the structures for specific spectral response. We demonstrate design and fabrication of large area quasicrystal air hole patterns of $\pi / 5$ symmetry in metal film in which broadband, polarization and launch angle independent transmission enhancement is observed. We demonstrate bi-grating quasicrystals to show that designable transmission response is possible over visible to near infrared wavelength regions with about 15 times enhancement. These would be useful in many applications like energy harvesting, nonlinear optics and quantum plasmonics.

ub-wavelength patterns in the form of grooves, hole arrays, or corrugations on metal-dielectric interfaces help optically excite surface plasmon polaritons (SPPs) at the interface ${ }^{1}$. SPPs enhance transmission as well as the local field and have wide ranging applications in nanophotonics ${ }^{2-12}$. Plasmonic crystals are periodic metal-dielectric structures realized for a specific application in which the SPPs are optically excited by periodic structures $^{1}$. The translation symmetry of these crystals in a given direction results in discrete SPP excitation. For example, the longitudinal SPP modes are excited by 1-dimensional patterns (gratings) by the transverse magnetic (TM) field though under specific orientations transverse electric field may excite SPPs ${ }^{13}$. In the case of 2 -d patterns with circular constituents (holes or discs), while for square lattice the TE and TM polarizations are equivalent due to $\pi / 2$ rotational symmetry, for non-square lattices and lattices with asymmetric shaped holes, unless specifically designed, the response will be different for different polarizations for non-normal incidence. In general, the projection of momentum onto the surface is given by $\mathrm{k}_{\mathrm{x}}{ }^{2}+\mathrm{k}_{\mathrm{y}}{ }^{2}=\mathrm{k}_{\mathrm{SPP}}{ }^{2}$, where $\mathrm{k}_{\mathrm{x}}=\mathrm{k}_{0} \sin (\theta) \cos (\varphi) \pm \mathrm{i} 2 \pi / \mathrm{a}_{\mathrm{x}}$ and $\mathrm{k}_{\mathrm{y}}=\mathrm{k}_{0} \sin (\theta) \sin (\varphi) \pm \mathrm{j} 2 \pi / \mathrm{a}_{\mathrm{y}}$ in which $\mathrm{i}, \mathrm{j}$ are integers, $\mathrm{a}_{\mathrm{x}}, \mathrm{a}_{\mathrm{y}}$ are lattice constants in the in-plane directions, $\theta$ is the angle of incidence and $\varphi$ is the azimuthal angle. It would be interesting if the dependence of the discrete SPP mode excitation on the orientation of the sample or the azimuthal angle $\varphi$ and thus polarization of light can be overcome $^{15,16}$. Unlike crystals, quasicrystals have only long range ordering and rotational symmetry ${ }^{14}$. In quasi crystal patterns, discrete SPP modes ${ }^{17}$, extra-ordinary transmission in different wavelength regions ${ }^{18-24}$, higherharmonic generation ${ }^{25,26}$, focusing and directivity of emission ${ }^{27,28}$ have been demonstrated. In addition to these designable k-space and broadband local field and transmission enhancement in plasmonic quasicrystals (PlQCs) would be useful. For example, for light harvesting, the top contact of the photovoltaic device can be patterned with quasicrystal structure, in nonlinear optics for harmonic generation though the metal surface optical nonlinearity is weak $\left(\chi^{(2)}\right)^{29}$, in quantum plasmonics for high quality factor cavities ${ }^{30}$. Though in random patterns one can achieve broadband response ${ }^{31}$, it is in practice not easy to design them for specific k-space response. Aperiodic structures and their applications to nanophotonics have been reported earlier ${ }^{32,33}$.

In order to design quasicrystal structures several methods based on tiling, grid, inflation/deflation, projection, decoration, cluster model, etc., are employed ${ }^{32,34}$. In most of the previous reports on PlQCs, the dual grid method or the Penrose tiling using fat and thin rhombus shaped tiles for designing and ion beam milling for patterning the thin metal film were employed ${ }^{18-28}$. Electron beam lithography has been used for making gold nanodots arranged in aperiodic patterns ${ }^{31}$ and Moire nanolithography has been used to demonstrate quasicrystal patterns of high rotational symmetry ${ }^{35}$. In optical domain, the effect of size of the pattern on the transmission enhancement was studied as well as the origin of transmission enhancement was proposed to be not related to long range ordering $^{18,19}$. Both these studies are on patterns covering an area of $10 \times 10 \mu \mathrm{m}^{2}$ milled in silver thin films and thus are 
comparable to or smaller than the propagation length of SPPs. We present a method to realize quasicrystal patterns over large area using electron beam lithography. The design and fabrication methods can be easily extended to any rotational symmetry and Penrose tiles. We present in PlQCs with $\pi / 5$ symmetry, polarization independent, near dispersionless broadband transmission enhancement compared to unpatterned metal. We demonstrate ultra broadband transmission enhancement in PlQCs.

\section{Results}

In oblique tiling method, a set of parallel lines tilted by $\pi / \mathrm{n}$ angle are superimposed by $n$ similar sets, each rotated by $\pi / \mathrm{n}$ with respect to the previous set. The intersection points of the $n$ sets of these parallel lines give the coordinates of the quasicrystal with n-fold symmetry. For generating the soft mask for electron beam lithography, the coordinates of the quasicrystal lattice points are needed. For this purpose, we modify the design method by replacing the continuous lines with dots and thus the set of parallel lines are now a 2-D array of dots as shown in Figure 1a. By successively superimposing $n$ such 2dimensional arrays of dots that are rotated by $\pi / \mathrm{n}$ with respect to the previous array, we generate a hole pattern. The spatial points which are common to all the $\mathrm{n}$ overlays give the coordinates of the $\mathrm{QC}$ of $\pi / \mathrm{n}$ rotational symmetry.

There are two main advantages of this design methodology. The intrinsic period of the starting 2-d array defines the center of the spectral response and thus can be tuned to any wavelength required. Thus, the spectral response of the quasicrystal pattern is designable. Secondly, this method simplifies the complexity in identifying the coordinates of the quasicrystal lattice points from the large set of points for generating the $\mathrm{CAD}$ for electron beam lithography. However, the repeated points need to be removed to avoid multiple exposures. To find repetitions in the large array of points, we used QuickSort algorithm which is a sort-in-place algorithm that does not need additional memory and sorts an array of size $\mathrm{N}$ in $\mathrm{O}(\mathrm{N} \times$ $\log (\mathrm{N}))$ time $^{36}$. This helps identify repetitions in approximately $\mathrm{O}(\mathrm{N})$ time which is much faster than the time taken by brute force methods $\left(\mathrm{O}\left(\mathrm{N}^{2}\right)\right)$.

The coordinates of the quasicrystal pattern are generated over an area of $\sim 1 \mathrm{~mm}^{2}$. By electron beam lithography and dry etching, we patterned thin gold film on optically flat quartz (see Methods). Fig. $1 b$ shows the SEM image of the fabricated quasicrystal. The initial rhombus used in designing the structure had $1 \mathrm{~mm}$ long sides each having holes at a periodic separation ( $a$ in Fig. 1a) of $600 \mathrm{~nm}$. That is, in each set, there are about 2.8 million points in $1 \mathrm{~mm}^{2}$ area and with 5 superimpositions there are a total of about 14 million points.
In the following we use PlQCs in which all the holes excepting those that are within $10 \mathrm{~nm}$ vicinity of a given hole are retained to avoid over exposure during e-beam lithography. This sorting results in, the coordinates of both QC of $\pi / 5$ symmetry as well as those corresponding to the 5 base lattices, about 13 million holes in $1 \mathrm{~mm}^{2}$ area. The average hole diameter obtained was $92 \pm 7 \mathrm{~nm}$ by careful e-beam dosage. The diffraction pattern (Fig. 1c) recorded using a $635 \mathrm{~nm}$ diode laser beam clearly shows $\pi / 5$ symmetry. The observed multiple concentric circles show that the structure displays ordering at different levels. Fig. 1d shows the calculated Fourier transform of the structure shown in Fig. 1a.

We performed angle resolved white light transmission measurements to measure the normalized transmission (ratio of transmission through patterned and unpatterned Gold) for TM, TE and unpolarized light for different $\theta$. Fig. 2(a) shows the measurement geometry and Fig. 2(b) compares the spectra for different polarizations for $\theta=$ $5^{\circ}$ and $\varphi=0^{\circ}$. Fig. 2(c) compares the transmission enhancement spectra for different $\theta$ and $\varphi$. Transmission spectra measured for different $\theta$ are plotted as contour plots for TE and TM polarizations in Fig. 2(d) and Fig. 2(e), respectively. Transmission dips correspond to SPP absorption and thus the dark contours show dispersion of various SPP modes. Similarly, bright regions correspond to transmission enhancement regions. We observe upto 9 fold transmission enhancement with respect to the unpatterned metal tranmission and near dispersionless broadband response seen as the featureless bright band between $550 \mathrm{~nm}$ and $720 \mathrm{~nm}$ wavelengths.

\section{Discussion}

To calculate the SPP resonances and their dispersion, for a periodic pattern, there are theoretical models for a given interface ${ }^{37,1}$. Analytical expressions for SPP dispersion for two layers (single interface) and three layers (two interfaces) are well known ${ }^{35}$. In order to calculate the grating excited SPP modes one can equate the 2 -d momentum conservation equation where appropriate $k_{S P P}$ is used depending on the layer structure. For example, for 2-d pattern of air holes ${ }^{38}$,

$$
\sqrt{\left(k_{0} \sin (\theta) \cos (\varphi) \pm \frac{m 2 \pi}{a_{1}}\right)^{2}+\left(k_{0} \sin (\theta) \sin (\varphi) \pm \frac{n 2 \pi}{a_{2}}\right)^{2}}=k_{S P P}
$$

where $\mathrm{a}_{1}$ and $\mathrm{a}_{2}$ are the periods in $\mathrm{x}$ and $\mathrm{y}$ directions, respectively. Compared to a periodic crystal, the azimuthal angle $(\varphi)$ dependence in a quasicrystal is negligible due to rotational symmetry. In addition, due to lack of translational symmetry, the Bloch periodic terms ( $\mathrm{m} 2 \pi$ ) $a_{1}$ and $n 2 \pi / a_{2}$ ) do not exist in the quasi crystals and are to be replaced with the reciprocal vectors which can be obtained from either the calculated $\mathrm{k}$-space picture or from measured diffraction patterns.

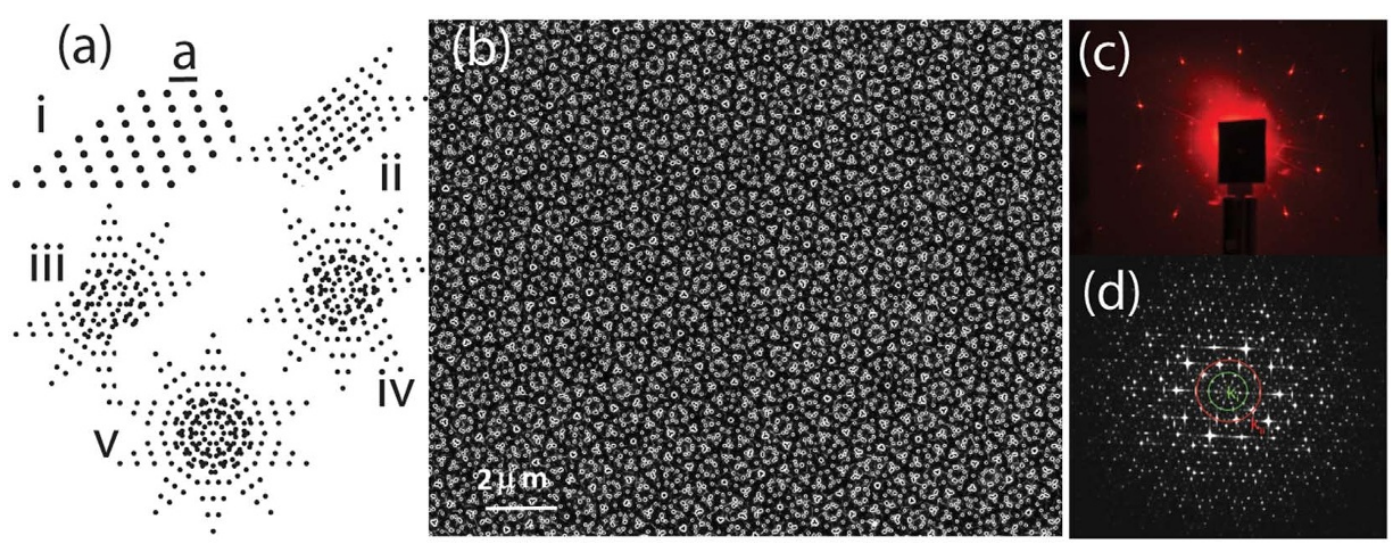

Figure $1 \mid$ (a) Schematic showing the steps in designing of quasicrystal with $\pi / 5$ rotational symmetry (see text for details). (b) SEM image of the

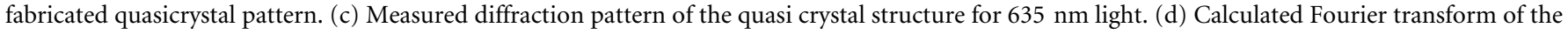
final pattern shown in (a). 

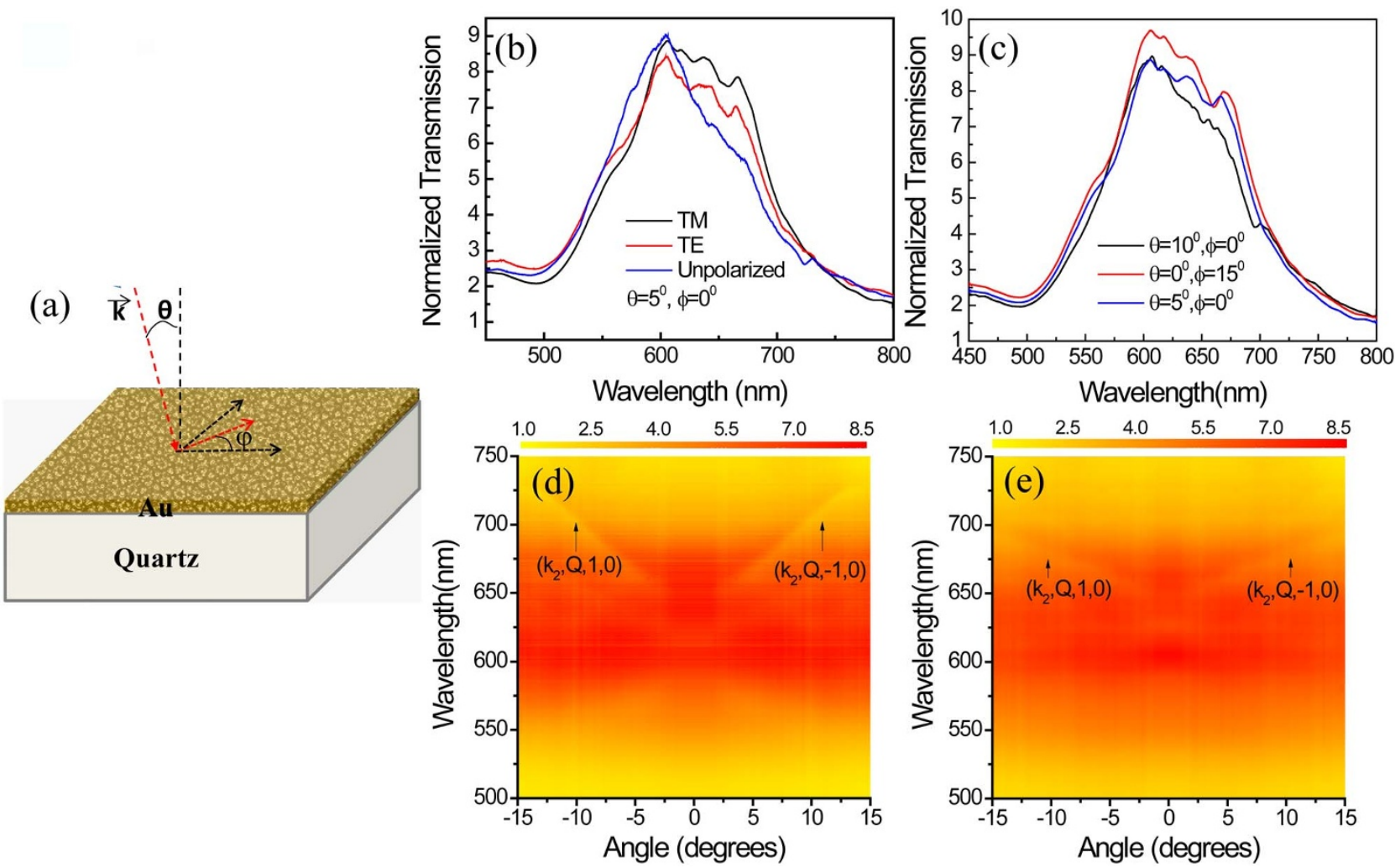

Figure $2 \mid$ (a) Schematic of the measurement geometry. Normalized transmission spectra are shown for different (b) incident polarizations and (c) $\theta$ and $\phi$ for TM polarized light. Measured launch angle dependence of the transmission enhancement is shown as contour plot for (d) TM and (e) TE polarizations. Top scale bar shows the transmission enhancement factor. Broadband transmission enhancement (red regions between $500-700 \mathrm{~nm}$ wavelength region) that is launch angle independent is seen. Some of the dispersive SPP modes are identified from the calculations.

Thus, the momentum conservation rule for QCs is given by ${ }^{17}, k_{\|}+$ $k_{S P P}=\mathrm{F}^{(\mathrm{i})}$ where $k_{\|}$and $k_{S P P}$ are the in-plane components of the wave vector of incident light and SPP, respectively and $\mathrm{F}^{(\mathrm{i})}$ are the reciprocal vectors. From the calculated Fourier transform of the pattern, one can extract the $\mathrm{F}^{(\mathrm{i})} \mathrm{s}$ and thus calculate the $k_{S P P} \mathrm{~s}$. We use this method in the following to calculate the SPP resonance positions which can, in principle, be used to calculate the expected spectral response. In practice, the dense $\mathrm{k}$-space makes it difficult to calculate the spectral response. Another practical advantage in calculating the SPP resonances is, if the calculated SPP resonances do not match the requirement, we can scale the pattern for specific k-response by using the scaling property of Fourier space which, for a constant $c$ and real and phase space functions given by $\mathrm{F}$ and $\mathrm{f}$, is given by, $\int_{-\infty}^{\infty} F(c k) e^{i k x} d k=\frac{1}{c} f\left(\frac{x}{c}\right)$.
We use the Fourier transform (FT) shown in Fig. 1d to deduce the reciprocal vectors $\left(\mathrm{k}_{\mathrm{I}}, \mathrm{k}_{\mathrm{II}}\right.$ etc.,) corresponding to the successive circles. For example, the values are $\mathrm{k}_{\mathrm{I}}=1.82 \mu \mathrm{m}^{-1}$ (red) and $\mathrm{k}_{\mathrm{II}}=$ $2.91 \mu^{-1}$ (green). Each SPP mode may be denoted in terms of a tuple $(\mathrm{K}, \mathrm{B}, \mathrm{m}, \mathrm{n})$. $\mathrm{K}$ takes the values $\mathrm{k}_{\mathrm{I}}, \mathrm{k}_{\mathrm{II}}, \mathrm{B}$ takes the values $\mathrm{Q}$ (quartz) or A (air) which defines the interface with gold, at which the SPP propagates and $(m, n)$ are integers. The calculated positions of various modes are marked in the measured SPP dispersion contour plots (Fig. 2(d) and Fig. 2(e)). For the calculations, the values of $\varepsilon_{\mathrm{m}}$ are taken from Johnson \& Christy parameters ${ }^{39}$ for gold and $\varepsilon_{\mathrm{d}}=1$, 2.25 for air and quartz, respectively. The resonance corresponding to $\mathrm{k}_{\mathrm{II}}$ is more prominent as can be seen in all the plots. This is consistent with the stronger diffraction maxima in Fig. $1 \mathrm{~d}$ for $\mathrm{k}_{\mathrm{II}}$ compared to $\mathrm{k}_{\mathrm{I}}$.

Fig. 2(b) shows the broadband transmission enhancement for TE, $\mathrm{TM}$ and unpolarized incident light for $\theta=5^{\circ}$ and $\varphi=0^{\circ}$.
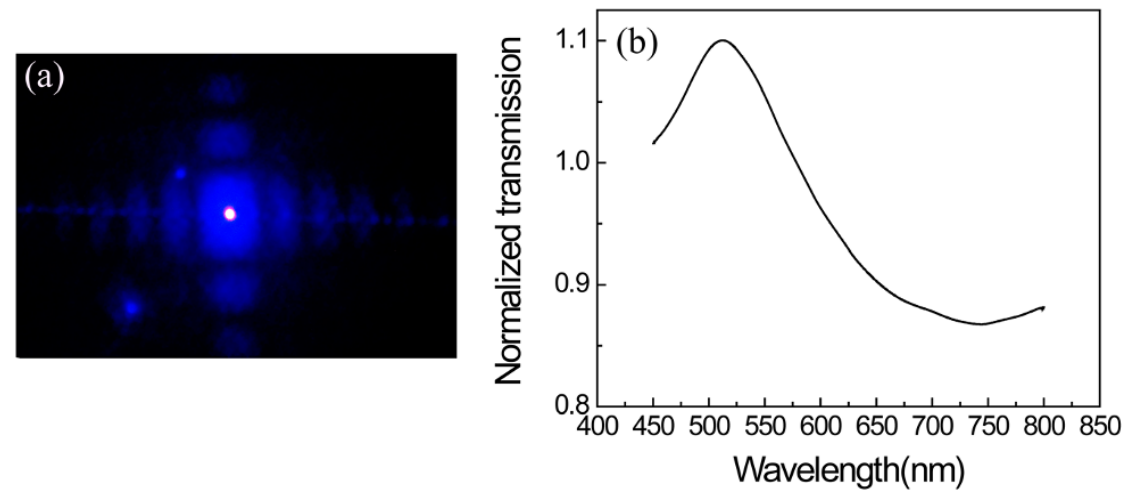

Figure $3 \mid$ (a) Measured diffraction pattern over a square lattice with identical hole density shows much sparser and weaker k-space compared to the quasicrystal pattern. (b) Shows the transmission enhancement spectrum for square lattice of air holes in gold. 


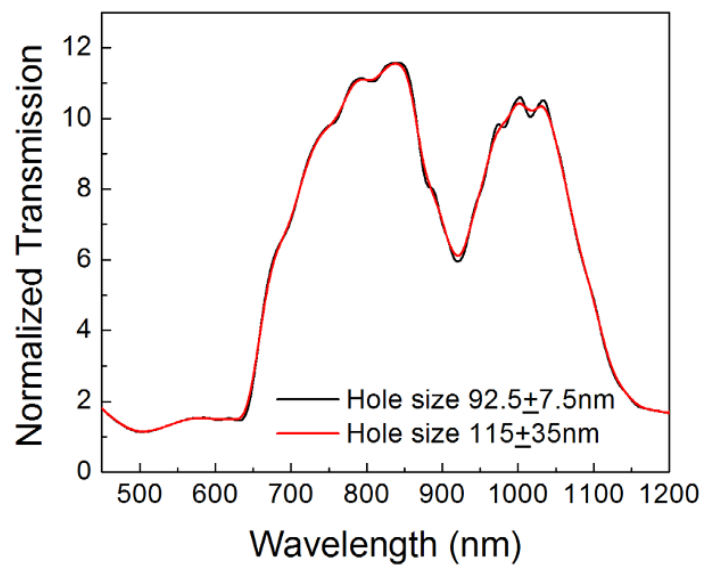

Figure $4 \mid$ A comparison of the transmission enhancement for two PlQCs of $1 \mu \mathrm{m}$ period with differing inhomogeneity in the air hole size shows very little dependence of spectral response on the hole inhomogeneity.

Polarization independence is reported to be a manifestation of rotational symmetry with no translational symmetry ${ }^{16}$. Orientation independence in the transmission enhancement is shown in Fig. 2(c) for different $\theta$ and $\varphi$ for TM polarized incident light. Measured launch angle dependent transmission enhancement spectra are plotted as a contour plot in Fig. 2(d) and 2(e) for TE and TM polarizations, respectively. Yellow contours corresponding to different SPP modes are identified from the calculations described above and are marked. $\mathrm{k}_{\mathrm{I}}$ mode is weak and the corresponding contours are not resolvable in the plots. Broad featureless red regions covering $500-700 \mathrm{~nm}$ wavelength range show the broadband transmission enhancement that is launch angle independent.

In addition, the multiple periodicities and dense k-space displayed could lead to greater number of SPP modes in the same wavelength range compared to 1-d or 2-d periodic structures, resulting in broad band response. Enhanced transmission is known to happen due to coupling of field from top to bottom. Thus, in addition to the pattern, metal thickness is also critical for efficient coupling and transmission enhancement. To rule out the role of high hole density (and fill factor) in the observed transmission enhancement, we tested a structure with 2-d square array of holes in metal with same metal thickness and with the same hole density (and thus fill factor) as the quasicrystal sample. Thus the square lattice has period of $150 \mathrm{~nm}$ with $85 \pm 10 \mathrm{~nm}$ diameter of holes. Measured diffraction pattern (Fig. 3(a)) with $442 \mathrm{~nm}$ laser is much sparser and weaker compared to the quasicrystal pattern indicating that we may observe narrow and weak transmission enhancement in the case of square lattice. Accordingly, we observed much lower transmission enhancement of $\sim 1.1$ compared to unpatterned metal for this structure as shown in Fig. 3(b). Sparse k-space and dispersive modes are already reported in square lattice patterns with period around $0.7 \mu \mathrm{m}^{38}$.

To check the role of inhomogeneity in the hole size, we compared two $1 \mu \mathrm{m}$ period quasicrystal structures with varying distribution in hole diameter. One structure has hole size distribution of $92.5 \pm$ $7.5 \mathrm{~nm}$ and the other has $115 \pm 35 \mathrm{~nm}$. Almost identical transmission behavior but with reduced resolution could be seen in Figure 4 as expected when inhomogeneity increases. It may be noted that the dip at about $950 \mathrm{~nm}$ observed in the spectrum is related to Wood's anomaly as has been predicted in quasicrystals ${ }^{40}$.

It is advantageous to have control over the design to realize PIQC structures with desired spectral response. This can have applications in light harvesting, filters, nonlinear optics and quantum plasmonics where manipulation of dipole emitters is needed. We show such feasibility in a dual grating PlQC realized by combining PlQCs with two different base periods of 0.6 and $1 \mu \mathrm{m}$ in which the transmission band has been increased to $800 \mathrm{~nm}$. To realize the bigrating, the $1 \mu \mathrm{m}$ base period QC pattern was superposed on $0.6 \mu \mathrm{m}$ base period QC pattern with a common center. Schematic of the individual patterns for the three QCs, $0.6 \mu \mathrm{m}, 1 \mu \mathrm{m}$ and the combined bigrating pattern, are shown in Fig. 5a, b, and c, respectively. Figure 5d shows the SEM image of the bigrating quasicrystal and Figure $5 \mathrm{e}$ shows the measured transmission enhancement curves for the three structures.
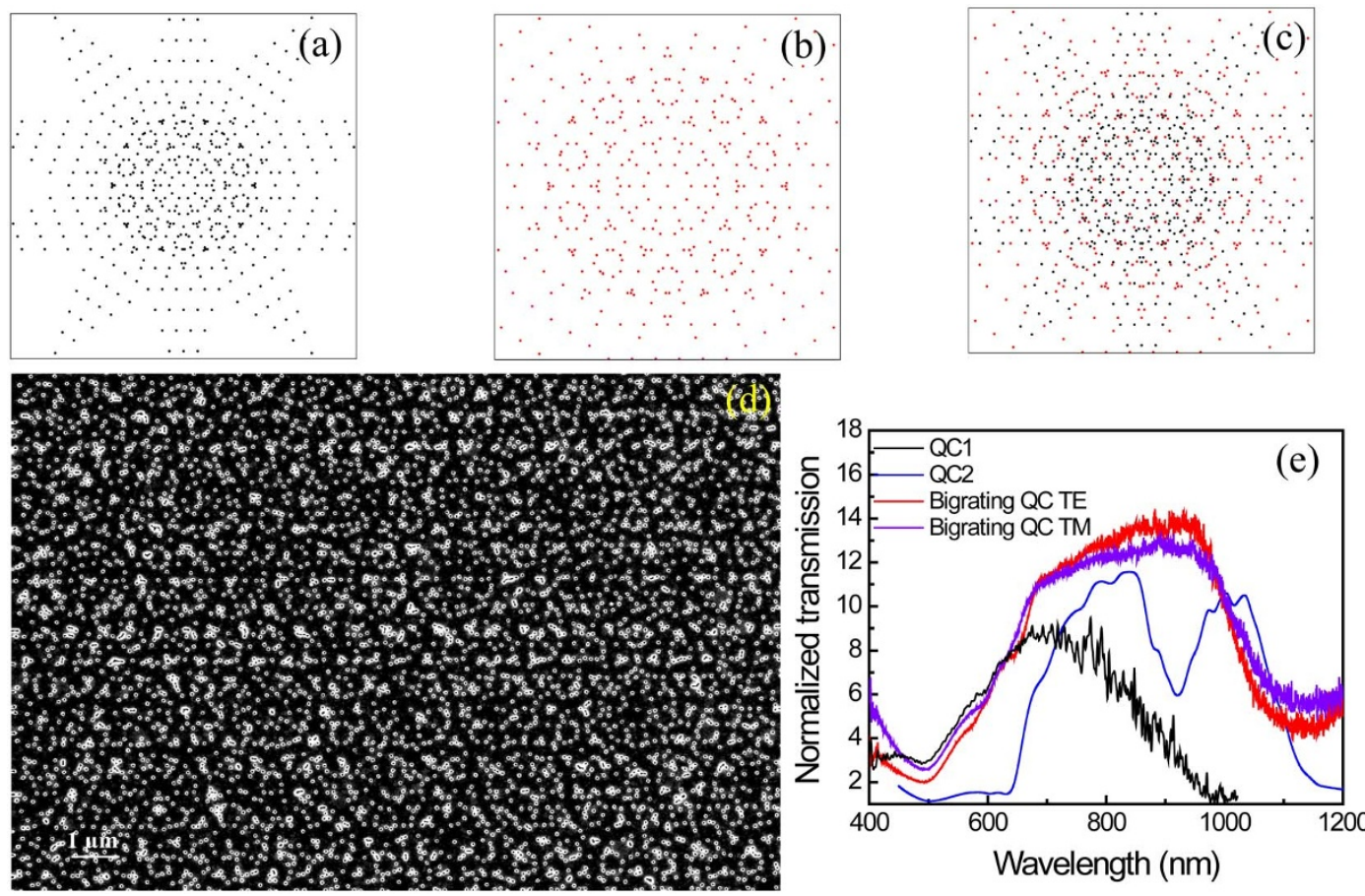

Figure $5 \mid$ Shown are the schematics of the quasicrystal patterns, QC1 and QC2, with period $0.6 \mu \mathrm{m}(\mathrm{a}), 1 \mu \mathrm{m}$ (b), respectively and the bigrating quasicrystal which is obtained by superposing QC1 and QC2 patterns with common center (c). SEM image of the bigrating quasicrystal is shown in (d) and transmission enhancement in single PIQCs and for TE and TM polarizations in the bigrating PlQC are shown in (e). 
Up to 15 times enhancement in transmission over a broad wavelength range could be seen for the bigrating structure for both TE and TM polarizations. Thus, multi-grating PIQCs could be a way forward to realize structures with required transmission response.

\section{Conclusion}

We demonstrated a method to design and fabricate large area quasicrystal patterns of a given rotational symmetry by electron beam lithography. As an example, we demonstrated $\pi / 5$ rotation symmetry plasmonic quasicrystals with clear optical diffraction pattern. We show in PIQCs with two different periods that the inherent lattice constant (or period) in the present method allows one to design the spectral response. These structures showed near dispersionless enhanced transmission over a broad wavelength range for both TE and TM polarizations. Also, the present method can be extended to multi-grating patterns to achieve broadband spectral response. We demonstrated one such structure with very broadband transmission enhancement of over an order of magnitude in bigrating PlQC compared to unpatterned gold film.

\section{Methods}

To prepare the sample, a $100 \mathrm{~nm}$ thick gold film was deposited in Argon (Ar) plasma by radio frequency (RF) magnetron sputtering on optically flat $1 \mathrm{~mm}$ thick quartz substrate. A polymer, electron beam resist, PMMA $495 \mathrm{~A} 4$, was spin coated on top of the gold film at $2000 \mathrm{rpm}$ to get a resist thickness of $200 \mathrm{~nm}$. Electron beam lithography (EBL) was then performed by using Raith e-line to pattern the resist with the designed quasicrystal pattern. A high density of holes meant that we had to use dose scaling to get good feature resolution. Subsequent development was done using MIBK:IPA (1:3) for 90 seconds followed by IPA treatment for 60 seconds. Reactive Ion Etching (RIE) was then used to Argon (Ar) sputter the exposed Gold regions to get air hole pattern in gold film. The Ar flow rate was $50 \mathrm{sccm}$ and chamber pressure was maintained at $1 \mathrm{~Pa}$. The radio frequency (RF) power used was $138 \mathrm{~W}$. Etching was done for 7 minutes to remove the gold. The resist was then stripped using $\mathrm{O}_{2}$ plasma at $80 \mathrm{~W}$ RF power at a flow rate of $50 \mathrm{sccm}$ for 6 minutes. To etch metals we modified the Sentech SI 500 inductively coupled plasma (ICP) RIE by placing a Teflon shield to cover and protect the ICP head. We reduced the damage of the gold surface by having 1-2 minute etch stop for every 1 minute etching.

For linear optical transmission, a $100 \mathrm{~W}$ tungsten halogen lamp was used as the white light source with multiple lenses and pinholes to collimate the beam to $<0.3^{\circ}$ divergence. A fiber coupled spectrometer (either Ocean Optics $\$ 4000$ with resolution $0.26 \mathrm{~nm}$ or $0.5 \mathrm{~m}$ Acton SP2500 spectrometer with thermoelectrically cooled PIXIS $400 \mathrm{BR}$ CCD with $0.05 \mathrm{~nm}$ resolution at $600 \mathrm{~nm}$ ) was used to collect and characterize the signal in the $450-1200 \mathrm{~nm}$ wavelength range. A Glan-Thompson polarizer (extinction ratio $10^{5}: 1$ ) was used to get linearly polarized light (TE or TM). To perform angle resolved measurements, a motorized rotation stage with $0.03^{\circ}$ angle resolution was used.

C-programs were written to create the design and extract the coordinates of the lattice points of the quasi crystal pattern as well as for QuickSort algorithm.

\section{Raether, H. Surface Plasmons on Smooth and Rough Surfaces and on Gratings} (Springer, 1986)

2. Ebbesen, T. W., Lezec, H. J., Ghaemi, H. F., Thio, T. \& Wolff, P. A. Extraordinary optical transmission through sub-wavelength hole arrays. Nature 391, 667-669 (1998)

3. Martín-Moreno, L. et al. T. W. Theory of Extraordinary Optical Transmission through Subwavelength Hole Arrays. Phys. Rev. Lett. 86, 1114-1117 (2001).

4. Belotelov, V. I. et al. Enhanced magneto-optical effects in magnetoplasmonic crystals. Nature NanoTech. 6, 370-376 (2011).

5. Belotelov, V. I. et al. Plasmon mediated magneto-optical transparency. Nature Commun. 4, 2128 (2013).

6. Zhang, J. Z. \& Noguez, C. Plasmonic optical properties and applications of metal nanostructures. Plasmonics. 3, 127-150 (2008).

7. Ma, R.-M., Oulton, R. F., Sorger, V. J., Bartal, G. \& Zhang, X. Room-temperature sub-diffraction-limited plasmon laser by total internal reflection. Nature Materials. 10, 110-113 (2011).

8. Tvingstedt, K., Persson, N.-K., Inganäs, O., Rahachou, A. \& Zozoulenko, I. V. Surface plasmon increase absorption in polymer photovoltaic cells. Appl. Phys. Lett. 91, 113514 (2007)

9. Barnes, W. L., Dereux, A. \& Ebbesen, T. W. Surface plasmon subwavelength optics. Nature 424, 824-830 (2003).

10. Righini, M., Zelenina, A. S., Girard, C. \& Quidant, R. Parallel and selective trapping in a patterned plasmonic landscape. Nat. Phys. 3, 477-480 (2007).

11. Zhang, X., Liu, H., Tian, J., Song, Y. \& Wang, L. Band-selective optical polarizer based on gold-nanowire plasmonic diffraction gratings. Nano Lett. 8, 2653-2658 (2008).
12. Antoine, R. et al. Surface plasmon enhanced second harmonic response from gold clusters embedded in an alumina matrix. J. Appl. Phys. 84, 4532-4536 (1998).

13. Vengurlekar, A. S. Polarization dependence of optical properties of metallo dielectric gratings with subwavelength grooves in classical and conical mounts. J. Appl. Phys. 104, 023109 (2008).

14. Penrose, The role of aesthetics in pure and applied mathematical research. R. Bull Inst. Math. Its Appl. 10, 266-271 (1974).

15. Popov, E., Maystre, D., McPhedran, R. C., Nivière, M., Hutley, M. C. \& Derrick, G. H. Total absorption of unpolarized light by crossed gratings. Opt. Express. 16, 6146-6155 (2008).

16. Bliokh, Y. P., Brodsky, Y. L., Chashka, Kh, B., Felsteiner, J. \& Slutsker, Y. Z. Broadband polarization-independent absorption of electromagnetic waves by an overdense plasma. Phys. Plasmas. 17, 083302 (2010).

17. Matsui, T., Agrawal, A., Nahata, A. \& Vardeny, Z. V. Transmission resonances through aperiodic arrays of subwavelength apertures. Nature 446, 517-521 (2007).

18. Przybilla, F., Genet, C. \& Ebbesen, T. W. Enhanced transmission through Penrose subwavelength hole arrays. Appl. Phys. Lett. 89, 121115 (2006).

19. Pacifici, D., Lezec, H. J., Sweatlock, L. A., Walters, R. J. \& Atwater, H. A. Universal optical transmission features in periodic and quasiperiodic hole arrays. Opt. Express. 16, 9222-9238 (2008).

20. Papasimakis, N., Fedotov, V. A., Schwanecke, A. S., Zheludev, N. I. \& Garci'a de Abajo, F. J. Enhanced microwave transmission through quasicrystal hole arrays. Appl. Phys. Lett. 91, 081503 (2007).

21. Hao, R., Jia, H., Ye, Y., Liu, F., Qiu, C., Ke, M. \& Liu, Z. Exotic acoustic transmission through hard plates perforated with quasiperiodic subwavelength apertures. Europhys. Lett. 92, 24006 (2010).

22. Agrawal, A., Matsui, T., Vardeny, S. V. \& Nahata, A. Terahertz transmission properties of quasiperiodic and aperiodic aperture arrays. J. Opt. Soc. Am. B. 24, 2545-2555 (2007).

23. Rockstuhl, C., Lederer, F., Zentgraf, T. \& Giessen, H. Enhanced transmission of periodic, quasiperiodic, and random nanoaperture arrays. Appl. Phys. Lett. 91, 151109 (2007).

24. Xue, J., Zhou, W.-Z., Dong, B.-Q., Wang, X., Chen, Y., Huq, E., Zeng, W., Qu, X.-P. \& Liu, R. Surface plasmon enhanced transmission through planar gold quasicrystals fabricated by focused ion beam technique. Microelectron. Eng. 86, 1131-1133 (2009).

25. Lifshitz, R., Arie, A. \& Bahabad, A. Photonic quasicrystals for nonlinear optical frequency conversion. Phys. Rev. Lett. 95, 133901 (2005)

26. Xu, T., Zhang, G. P. \& Blair, S. Second harmonic emission from sub-wavelength apertures: Effects of aperture symmetry and lattice arrangement. Opt. Expr. 15, 13894-13906 (2007).

27. Huang, F. M., Zheludev, N., chen, Y. \& Garcia de Abajo, F. J. Focusing of light by a nanohole array. Appl. Phys. Lett. 90, 091119 (2007).

28. Regan, C. J., Grave de Peralta, L. \& Bernussi, A. A. Directivity and isotropic bandgap in 12-fold symmetry plasmonic quasicrystals with small index contrast. Appl. Phys. Lett. 99, 181104 (2011).

29. Jha, S. S. Theory of optical harmonic generation at a metal surface. Phys. Rev. 140, A2020-A2030 (1965)

30. Min, B., Ostby, E., Sorger, V., Ulin-Avila, E., Yang, L., Zhang, X. \& Vahala, K High-Q surface-plasmon-polariton whispering gallery microcavity. Nature 457, 455-458 (2009)

31. Gopinath, A., Boriskina, S. V., Feng, N.-N., Reinhard, B. M. \& Negro, L. D. Photonic-plasmonic scattering resonances in deterministic aperiodic structures. Nano Lett. 8, 2423-2431 (2008).

32. Macia, E. Exploting aperiodic designs in nanophotonic devices. Rep. Prog. Phys. 75, 036502 (2012).

33. Vardeny, Z. V., Nahata, A. \& Agrawal, A. Optics of photonic quasicrystals. Nature Photonics 7, 177-187 (2013)

34. Socolar, J. E. S., Steinhardt, P. J. \& Levine, D. Quasicrystals with arbitrary orientational symmetry. Phys. Rev. B. 32, 5547-5550 (1985).

35. Lubin, S. M., Zhou, W., Hryn, A. J., Huntington, M. D. \& Odom, T. W. High rotational symmetry lattices fabricated by Moire nanolithography. Nano Lett. 12, 4948-4952 (2012).

36. Cormen, T. H., Leiserson, C. E., Rivest, R. L. \& Stein, C. Introduction to Algorithms (MIT Press, McGraw Hill Book Company, 2001).

37. Maier, S. A. Plasmonics: Fundamentals and Applications (Springer, 2007)

38. Kasture, S. et al. Near dispersion-less surface plasmon polariton resonances at a metal-dielectric interface with patterned dielectric on top. Appl. Phys. Lett. 101, 091602 (2012).

39. Johnson, P. B. \& Christy, R. W. Optical constants of the noble metals. Phys. Rev. B. 6, 4370-4379 (1972).

40. Bravo-Abad, J., Fernández-Domínguez, A. I., García-Vidal, F. J. \& MartínMerano, L. Theory of extraordinary transmission of light through quasiperiodic arrays of subwavelength holes. Phys. Rev. Lett. 99, 203905 (2007).

\section{Author contributions}

V.G.A. conceived the idea. S.K. developed the design methodology. S.K., A.P.R. and G.M. fabricated the samples. S.K., A.P.R., V.J.Y., R.P., N.V.V. did the measurements. V.G.A. wrote the manuscript with contributions from all. 


\section{Additional information}

Competing financial interests: The authors declare no competing financial interests.

How to cite this article: Kasture, S. et al. Plasmonic quasicrystals with broadband transmission enhancement. Sci. Rep. 4, 5257; DOI:10.1038/srep05257 (2014). cc) (i) $\Theta$ This work is licensed under a Creative Commons Attribution-NonCommercialNoDerivs 4.0 International License. The images or other third party material in this article are included in the article's Creative Commons license, unless indicated otherwise in the credit line; if the material is not included under the Creative Commons license, users will need to obtain permission from the license holder in order to reproduce the material. To view a copy of this license, visit http:// creativecommons.org/licenses/by-nc-nd/4.0/ 